\title{
The design and implementation of automotive radar system based on MFSK waveform
}

\author{
Yong Kun SONG*, Yong Ji LIU, Zhan Long SONG. \\ School of Information and Communication, Guilin University of Electronic Technology, Guilin, 541004, China
}

\begin{abstract}
As a driving aid system, automotive radar plays a more and more important role in reducing traffic accidents. In this paper, a simple and efficient automotive radar system based on MFSK (Multiple frequency shift keying) is designed, the system structure and implementation algorithm is presented. This system uses FPGA as the signal processing unit to ensure the response speed. In addition, the designed MFSK waveform algorithm achieved high precision and unambiguous measurement. The effectiveness of the system is verified by experiments and field measurements.
\end{abstract}

\section{Introduction}

The key technical challenge and problem of automotive radar system is how to get accuracy range, radial velocity and azimuth of multiple targets simultaneously, where the design of waveform plays an important role to solve those problems. The most commonly used LFM (Linear frequency modulation) waveform radar provides high range and velocity resolution, but it can not solve the problem of ghost targets in multiple targets situation. In addition, the radar system of FSK (Frequency shift keying) waveform has high velocity resolution and can avoid the ghost targets, but it is unable to measure the stationary target and multiple targets with the same radial velocity $[1,2,3]$.

In order to solve these problems, many improved methods have been proposed. In [4,5], a variable-period trapezoidal wave radar system is studied, the system introduced a constant frequency on the basis of the traditional upper and lower sweep frequency, which can reduce the number of ghost targets. However, the complex range and velocity matching algorithm greatly increase the amount of calculation. Paper [6] proposed a period-varying sawtooth wave measurement method, but the method with minimum coupling range difference may loss the target with large Doppler shift and generate ghost targets. An adaptive beam-controlled phased array radar is designed to improve the measuring distance and precision, but the complicated hardware structure and high cost limit its application [7,8,9].

An automotive radar system with MFSK waveform is designed in this paper, which is implemented based on FPGA. This system overcomes the shortcoming of the LFM and FSK system, it combines the advantage of the two systems, provides high range and velocity resolution, short measurement time and also could avoids ghost targets [10]. Firstly, this paper analyzed the range and velocity measurement theory of MFSK radar system, which filled up the previous theoretical blank, then designed the corresponding system structure and FPGA implementation algorithm. The filed experiment results show that the system has the fast response time and high measurement accuracy.

\section{Measurement Principle Analysis}

This system uses MFSK waveform as the modulation signal, which is composed of LFM and FSK signals. The transmitted and received signals are shown in the Fig.1. A periodic transmitted signal consists of two alternate and stepwise modulation signals, A and B wave. The real line is transmitted signal and the dotted line is received signal.

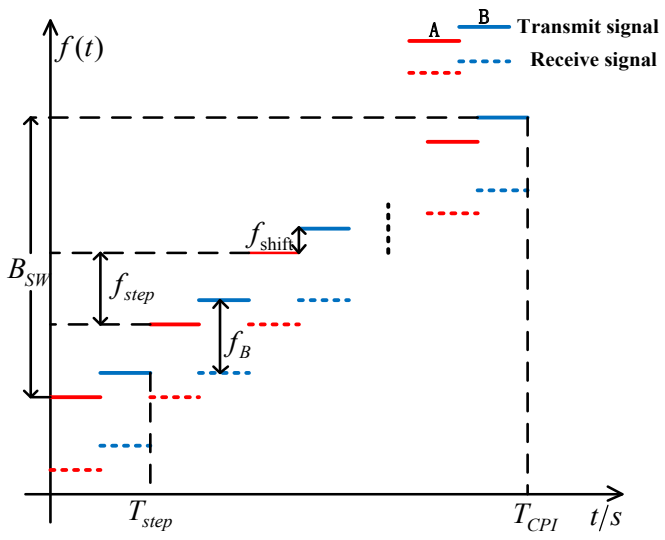

Fig. 1 MFSK transmitted and received waveform

Based on the characteristics of MFSK waveform, the radar system measurement principle can be analyzed.

Set $\mathrm{N}$ frequency transmitted signals as:

$x_{T}(i)=A_{T} \cdot \exp \left\{-j 2 \pi\left(f_{0}+i \cdot f_{\text {step }}\right) \cdot t\right\} \quad i=1,2 \ldots N-1$

The received signal is:

$$
x_{R}(i)=A_{R} \cdot \exp \left\{-j 2 \pi\left(f_{0}+i \cdot f_{\text {step }}\right) \cdot(t-\tau(i))\right\}
$$

\footnotetext{
* Corresponding author: songyk_1993@163.com
} 
After down-conversion, the difference frequency signal is simplified as follows:

$$
x_{s}(i)=A_{s} \cdot \exp \left\{-j 2 \pi\left(f_{0}+i \cdot f_{\text {step }}\right) \cdot \tau(i)\right\}
$$

where $\tau(i)=2\left(R+v i T_{\text {step }} / 2\right) / C$.

the $\mathrm{N}$ difference frequency signals are transformed by FFT, and it can be obtained that:

$$
\begin{aligned}
X(k)= & A_{s} \cdot \exp \left(-j \frac{4 \pi}{C} f_{0} R\right) \cdot \exp \left(-j 2 \pi \frac{N-1}{2} \cdot F\right) \\
& \cdot \sum_{i=0}^{N-1} \exp \left(\frac{-j 4 \pi v i^{2} f_{\text {step }} T_{\text {step }}}{2 C}\right) \cdot \frac{\sin (\pi N \cdot F)}{\sin (\pi \cdot F)}
\end{aligned}
$$

Where $F=\left(v f_{0} T_{\text {step }}+2 R f_{\text {step }}\right) / C+k / N$, it can be known that $X(k)$ pick up the peak of the spectrum when $k=-N\left(v f_{0} T_{\text {step }}+2 R f_{\text {step }}\right) / C$. Meanwhile $T_{\text {step }}=T_{C P I} / N$ and $f_{\text {step }}=B_{S W} / N$, so the beat frequency can be obtained.

$$
f_{B}=\frac{k}{T_{C P I}}=-\frac{2 \cdot v}{\lambda}-\frac{2 \cdot R \cdot B_{S W}}{C \cdot T_{C P I}}
$$

For equation (4), when $X(k)$ get to the peak, the phase of its peak is $\varphi=-4 \pi f_{0} R / C$, in addition, $R_{B}=R_{A}+v \cdot T_{\text {step }} / 4$, which results that the phase difference is as follows:

$$
\Delta \varphi=\varphi_{B}-\varphi_{A}=-\frac{\pi \cdot v}{(N-1) \cdot \Delta v}-4 \cdot \pi \cdot R \cdot \frac{f_{\text {shift }}}{C}
$$

With the two related equation (5) and (6), the target range and velocity can be resolved simultaneously. It can be seen that $f_{B}$ and $\Delta \varphi$ are one-to-one correspondence, there is no cross aliasing. Therefore, the MFSK system can effectively avoids ghost target problems which exist in LFM system. Moreover, the MFSK waveform can get a large number of frequencies and a wide range of span, as it is composed of multiple stepwise FSK signals. Different targets are reflected by different frequency levels to obtain different IF(Intermediate Frequency) signals, which overcomes the defects that FSK system can not measure multiple targets with the same radial velocity since it only has two frequencies .

\section{System Design}

The hardware structure of MFSK automotive radar system consists of four parts is shown in Figure 2.

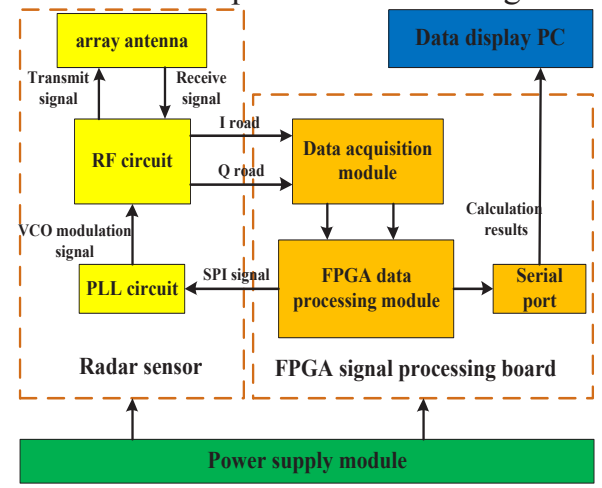

Fig. 2 Block diagram of MFSK automotive radar system
This system includes power supply module, radar sensor, FPGA signal processing board and data display PC. The power supply module provides the appropriate voltage and current for the system. The radar sensor includes $24 \mathrm{GHz}$ array antenna, RF (Radio Frequency) circuits and PLL (Phase-locked loop) circuit, it is responsible for signal emission and reception. The FPGA signal processing board includes data acquisition module, FPGA data processing module and serial port, which is mainly used for the signal processing and data transmission of the entire system. The data display PC is used to display the measurement results.

\section{FPGA Signal Processing Algorithm}

As the core of this system, FPGA controls the timing sequence, logic and signal processing of the whole system. The internal signal processing block diagram is shown in Figure 3 as below.

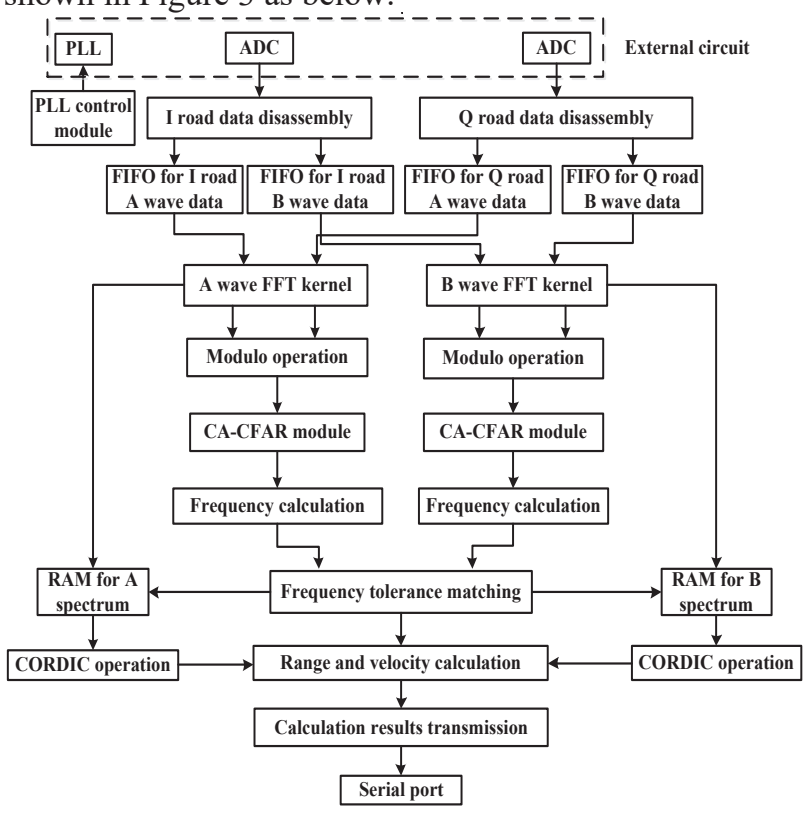

Fig. 3 FPGA internal signal processing block diagram

Firstly, the SPI(Serial Peripheral Interface) signal controls the PLL circuit of the radar sensor to generate the high precision MFSK signal, which could improve the modulation accuracy, then FPGA controls the ADCs to transform the orthogonal I and Q road signals into digital data. According to the measurement principle in section 2, the corresponding echo signals of $\mathrm{A}$ and $\mathrm{B}$ needs to be processed separately. The receiving orthogonal I and Q road data will be disassembled at the end of each short frequency step, after that the data are temporarily stored in the corresponding FIFO. Then the data are respectively transformed by FFT kernel, the transformed spectrum data is stored in RAM. At the same time, the absolute value of the spectrum data is obtained by modulo operation, then the data will be processed by the CA-CFAR module. This module can not only suppress system noise, but also effectively improve the detection efficiency of the spectrum peaks and reduce the system response time.

Then the data are respectively passed through the frequency calculation module to calculate the 
corresponding frequencies of the spectrum. Since the frequencies of the $\mathrm{A}$ and $\mathrm{B}$ wave which corresponding to the same target should be equal, due to the systematic error, the final calculation result may be little different. A frequency matching tolerance function is designed: $\left|f_{A k}-f_{B k}\right| \leq 2 / T_{C P I}$, where is the modulation period of the MFSK signal. If the A and B frequency values satisfy this function, it means that the frequency corresponds to the same target, the target peak position and the frequency value can be obtained. According to the location of the peak points, the spectrum raw data of the corresponding location are extracted from A road RAM and B road RAM, then the data is sent to CORDIC (Coordinate Rotation Digital Computer) module to calculate the phase like equation (7).

$$
\varphi=\arctan \left(\frac{I(t)}{Q(t)}\right)
$$

In this system, an improved CORDIC algorithm is used to perform the inverse tangent operation, and the data compensation is carried out too. The floating point number is converted to the fixed point number to improve calculation precision. The phase difference is gained by subtracting the phase values of A and B wave, which is used to calculate the range and velocity of the target with the frequency obtained before. Finally, FPGA sends the results to external device through the serial port.

\section{The FPGA Implementation And Field Experiment}

The parameters of the system designed in this paper are as Table 1 .

Table 1. System parameters

\begin{tabular}{|c|c|}
\hline Parameter & Value \\
\hline Center frequency & $24 \mathrm{GHz}$ \\
\hline Sweep bandwidth & $150 \mathrm{MHz}$ \\
\hline Modulation period & $100 \mathrm{~Hz}$ \\
\hline Step number & 100 \\
\hline Step frequency & $1500 \mathrm{KHz}$ \\
\hline Step time & $0.1 \mathrm{~ms}$ \\
\hline Range resolution & $1 \mathrm{~m}$ \\
\hline Velocity resolution & $0.625 \mathrm{~m} / \mathrm{s}$ \\
\hline Maximum range & $80 \mathrm{~m}$ \\
\hline
\end{tabular}

In this system, the PLL chip is ADF4158, which is a $6.1 \mathrm{GHz}$ fractional $\mathrm{N}$ frequency synthesizer with modulation and waveform generating capacity. Moreover, FPGA signal processing unit is EP4CE115F484. The system clock is $50 \mathrm{MHz}$, and the sampling clock is $200 \mathrm{KHz}$. 2048 points can be sampled in a modulation period, and the number of FFT point is 1024. In addition, the FPGA implementation language is Verilog HDL.

In order to test the performance of the system, some field experiments and matlab simulation are to be made, which measure the target of different motion state in the case of single target and multiple target. The whole system appearance and system filed test scene are shown in Fig.4, and the real time measurement results can be seen on the data display PC. It's like the results of the measurements shown in Fig. 4 , range is $15 \mathrm{~m}$, velocity is -

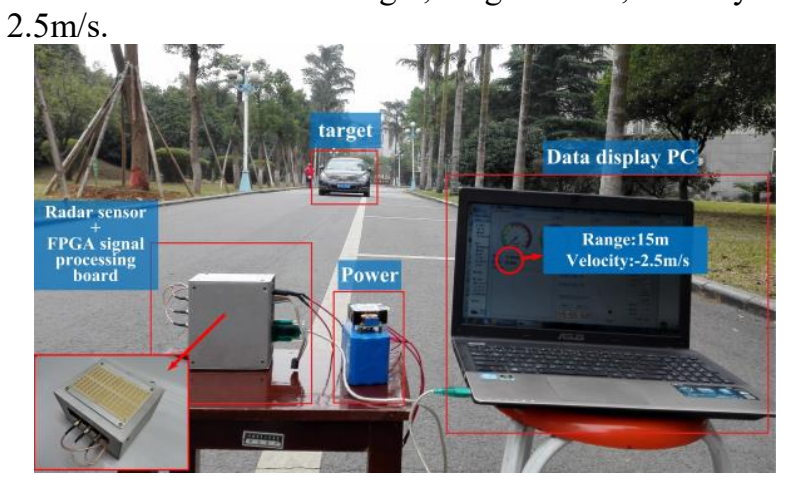

Fig. 4 System field test scene

\subsection{Single Target Field Measurement}

The experiment set single target as measurement object, which includes the stationary target and the moving target. In order to see the process of signal processing more intuitively, the real-time data is extracted and processed by matlab with the algorithm designed in section 4. One of the real-time data processing results are shown in Fig.5, it is the frequency and amplitude spectrum of A and B wave. Meanwhile, the frequency and phase value of the peaks are marked in the figure. As we can see from Figure 6, the frequencies of the two signals of A and B are basically equal, which represent that they are the echo of the same target, but the phase value is different. So the beat frequency and phase difference can be obtained. Moreover, the range and velocity can be calculated simultaneously.
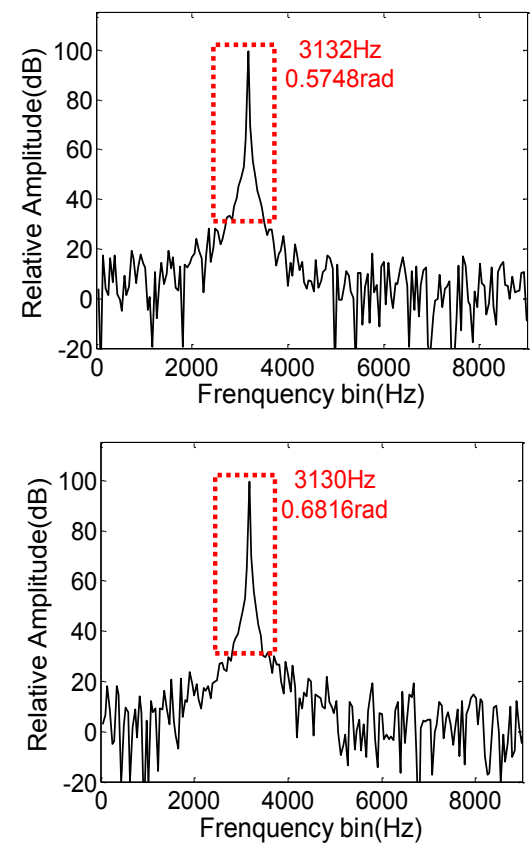

Fig.5 Single target data processing result, A wave(up), B wave(down) 
Two hundred groups of experiments were done in total, and two hundred targets are measured, the measurement results are recorded from the data display $\mathrm{PC}$, and the results are count as follow Table 2.

Table 2 Single target field measurement result statistics

\begin{tabular}{|c|c|c|c|c|}
\hline $\begin{array}{c}\text { Range } \\
\text { RMSE }\end{array}$ & $\begin{array}{c}\text { Velocity } \\
\text { RMSE }\end{array}$ & $\begin{array}{c}\text { Ghost target } \\
\text { number }\end{array}$ & $\begin{array}{c}\text { Lost target } \\
\text { number }\end{array}$ & $\begin{array}{c}\text { response } \\
\text { time }\end{array}$ \\
\hline $2.5 \%$ & $3 \%$ & 0 & 0 & $30 \mathrm{~ms}$ \\
\hline
\end{tabular}

\subsection{Multiple Target Field Measurements}

The multiple target measurement experiment to measure the multi-state target, which include stationary targets and moving targets, the targets with different velocity at the same range, the targets with different range at the same velocity, and the distinguishable target and non distinguishable target. Fig. 6 shows one of the real-time data processing results, it can be seen that the three frequencies of the $\mathrm{A}$ and $\mathrm{B}$ wave correspond to each other, which represent three different targets respectively. After frequency matching and phase calculation, the three targets' beat frequency and phase difference can be obtained, and then get the target motion information.
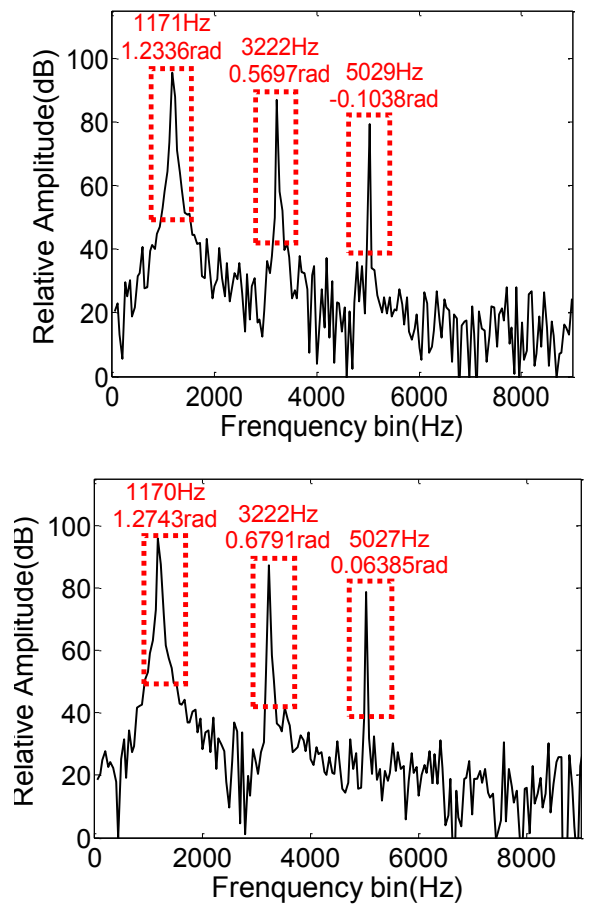

Fig.6 Multiple target data processing result, A wave(up), B wave(down)

The experiment also made two hundred groups field test, each group contains 2-4 targets, 500 targets are measured in total. The measurement result statistics as Table 3.

Table 3 Multiple target field measurement result statistics

\begin{tabular}{|c|c|c|c|c|}
\hline $\begin{array}{c}\text { Range } \\
\text { RMSE }\end{array}$ & $\begin{array}{c}\text { Velocity } \\
\text { RMSE }\end{array}$ & $\begin{array}{c}\text { Ghost target } \\
\text { number }\end{array}$ & $\begin{array}{c}\text { Lost target } \\
\text { number }\end{array}$ & $\begin{array}{c}\text { response } \\
\text { time }\end{array}$ \\
\hline
\end{tabular}

\begin{tabular}{|l|l|l|l|l|}
\hline $3 \%$ & $3.5 \%$ & 0 & 0 & $35 \mathrm{~ms}$ \\
\hline
\end{tabular}

\subsection{Experimental results analysis}

After massive experiments and field tests, the single target and multiple target measurement results are shown in Table II and table III. It illustrate that the system designed in this paper has high measurement accuracy, the measurement RMSE (Root Mean Square Error) of range and velocity is very small. Moreover, this system has little ghost targets in multi-target condition, and it can achieve unambiguous measurement even in the case of multiple targets with the same radial velocity. In addition, this system never lost target, and it also has fast response time.

\section{Conclusion}

In this paper, a FPGA-based MFSK automotive radar system is designed, the architecture of the system and the FPGA implementation algorithm are introduced in detail. At last, the system performance is proved by field test. The results show that MFSK method is better than traditional LFM and FSK methods, the FPGA implementation provides fast processing speed and high measurement accuracy. So the new system has good application prospect in real.

However, the automotive radar system designed in this paper has good measurement accuracy and fast response time under experimental conditions. There are no extra objects which affect the test, and the scene is relatively wide. In the case of real traffic road, the environment is more complex, and the system needs to be perfected continuously to reach the goal of adapting to more complex environment.

\section{References}

1. Patole S M, Torlak M, Wang D, et al. Automotive radars: A review of signal processing techniques[J]. IEEE Signal Processing Magazine, 2017, 34(2): 2235.

2. Macaveiu A, Nafornita C, Isar A, et al. A method for building the range-Doppler map for multiple automotive radar targets[C]. Electronics and Telecommunications (ISETC), 2014 11th International Symposium on. IEEE, 2014: 1-6.

3. Rohling $\mathrm{H}$, Moller $\mathrm{C}$. Radar waveform for automotive radar systems and applications[C]. Radar Conference, 2008. RADAR'08. IEEE. IEEE, 2008: 1-4.

4. Ziran X, Dongchen Z, Xing J. An effective method for multi-target range and velocity measurement in FMCW radar[J]. Acta Electronica Sinica, 2016, 44(9): 2148-2157.

5. Geroleo F G, Brandt-Pearce M. Detection and estimation of LFMCW radar signals[J]. IEEE Transactions on Aerospace and Electronic Systems, 2012, 48(1): 405-418. 
6. Kronauge M, Rohling H. New chirp sequence radar waveform[J]. IEEE Transactions on Aerospace and Electronic Systems, 2014, 50(4): 2870-2877.

7. Dudek M, Nasr I, Bozsik G, et al. System analysis of a phased-array radar applying adaptive beamcontrol for future automotive safety applications[J]. IEEE Transactions on Vehicular Technology, 2015, 64(1): 34-47.

8. Hu C, Liu Y, Meng H, et al. Randomized switched antenna array FMCW radar for automotive applications[J]. IEEE Transactions on Vehicular Technology, 2014, 63(8): 3624-3641.

9. Lee M S, Kim Y H. Design and performance of a 24-GHz switch-antenna array FMCW radar system for automotive applications[J]. IEEE Transactions on Vehicular Technology, 2010, 59(5): 2290-2297.

10. Rohling H. Development milestones in $24 \mathrm{GHz}$ automotive radar systems[C]. Radar Symposium (IRS), 2010 11th International. IEEE, 2010: 1-5. 